\title{
Snoring and Obstructive Sleep Apnea Syndrome: A reflection on the role of Dentistry in the current scientific scenario
}

\author{
Ângela Jeunon de Alencar e Rangel', Vinícius de Magalhães Barros², Paulo Isaias Seraidarian ${ }^{3}$
}

Introduction: Finally the dentist has awaken to the fact that by being a health professional, he has as primary function to take good care of the welfare of patients. In face of this challenge, the dentist starts to understand his role in the treatment of snoring and of obstructive sleep apnea and hypopnea.

Objective: The current paper has the purpose of discussing the role of this professional in the diagnosis and treatment of these diseases, most specifically of the therapy involving inter-occlusal devices, emphasizing the importance of multidisciplinarity in the reestablishment of the quality of life of the patient.

Keywords: Snoring. Obstructive sleep apnea and hypopnea. Occlusal plates.

\section{INTRODUCTION}

Among all sleep disorders, Obstructive Sleep Apnea-Hypopnea Syndrome (OSAHS) is the most prevalent one, diagnosed in $67.8 \%$ of the individuals refered to 19 centers of sleep evaluation. ${ }^{1}$ The OSAHS is a chronic disease, progressive and disabling, characterized by partial or total obstruction of the upper airway during sleep. ${ }^{2}$ In middle-aged individuals the prevalence is $2 \%$ to $4 \%,{ }^{3}$ more frequently seen in men, reaching 10 to $20 \%$ of them. ${ }^{4}$ Excessive daytime sleepiness, snoring,

${ }^{1}$ DDS, Post-Graduation student in Occlusion, Pain and Temporomandibular joint disorder, PUC-Minas.

${ }^{2}$ MSc in Dentistry, Emphasis in Prosthodontics, PUC-Minas.

${ }^{3}$ Coordinator of the MSc course in Dental Clinics, Emphasis in Dental Prosthesis, PUC-Minas. PhD in Restorative Dentistry, UNESP. MSc in Bucomaxilofacial Prosthesis. respiratory pauses, restless sleep with multiple micro-awakenings, morning headache, neurocognitive deficits, personality changes, reduced libido, depression and anxiety are common symptoms of this disease, causing emotional, social, occupational and marital damage. ${ }^{5}$

Even though its impact in public health may be overestimated, there are evidences of the association between hypertension ${ }^{6}$ and OSAHS, $\mathrm{CVD}^{7}$ and greater risks of car accidents. ${ }^{8}$ As for its progressive character,the treatment of this syndrome is not

How to cite this article: Alencar e Rangel AJ, Barros VM, Seraidarian PI. Snoring and Obstructive Sleep Apnea Syndrome: A reflection on the role of Dentistry in the current scientific scenario. Dental Press J Orthod. 2012 May-June;17(3):58-63.

\footnotetext{
Submitted: October 22, 2007 - Revised and accepted: November 19, 2010

» The authors report no commercial, proprietary or financial interest in the products or companies described in this article.

» Patients displayed in this article previously approved the use of their facial and intraoral photographs.

Contact address: Ângela Jeunon de Alencar e Rangel

Av. Prudente de Morais, 901 - Sala 802 - Santo Antônio, Belo Horizonte/MG - Brazil

Zip code: 30.380-000 - E-mail: ajeunonr@ig.com.br
} 
indicated only by the relief of symptoms, but also to decrease the risk of death ${ }^{9}$ and also by the savings of resources spent with health services. ${ }^{10}$

Dentistry is living a new era and crossing new frontiers, studying disciplines related to the overall health of the individual, highlighting the need of knowledge about sleep and its influence upon health and quality of life of individuals. Every dental surgeon plays an important role in identifying patients with sleep disorders, particularly snoring and OSAHS. Therefore, it seems to us, that a reflection on the role of dentistry is necessary for the diagnosis and treatment of this disease in the current scientific context.

\section{CONCEPTS AND PATHOPHYSIOLOGY OF SNORING AND SLEEP APNEA}

Snoring is a sign of different disorders. It's originated from the partial collapse of the tissues involved in the passage of air through the upper airway. A muscular tonus change in this region, results in a failure of maintaining the proper space for the airflow, specially in the deepest stages of sleep, is an important cause of snoring in adults. Unfortunately, this inappropriate muscular tonus is not very evident when in vigil. Tissue masses that obstruct the airflow, such as the increase in volume of the tonsils and adenoids, cysts, tumors, anatomical changes, as retro and micrognathia, nasal septum deformities, sinusitis and polyps are factors to be considered in the collapse of the upper airways. The fat accumulation in the neck region is relevant in breathing obstruction, meanwhile, a large cervical circumference is, by itself, an important data for the diagnosis of snoring. Similarly, conditions such as Down's syndrome and acromegaly, that are able to increase tongue size, also contribute to the presence of snoring. The restriction of the airflow through the nose increases the negative pressure during inspiration, causing partial collapse of the passage of the air flow. This would explain the common observation of people that usually do not snore, shall do so when they have flu or an allergy crisis. ${ }^{11}$

All airflow disruption that lasts two complete respiratory cycles is called apnea. The hypopnea is identified as the partial obstruction of more than $50 \%$ of the air flow.
Both can present variable lengths between 10 to 50 seconds. The OSAHS is classified according to the number of apnea episodes per hour: Slight (from 5 to 15 episodes of apnea/hour), moderate (from 15 to 35 episodes of apnea/hour) or severe (over 30 episodes of apnea/hour), the occurrence of up to 5 events per hour is considered normal. ${ }^{12}$ It must be pointed out that the central apnea mediated by the central nervous system, under no circumstances, can be treated as obstructive, the polysomnographic examination, so far, is the diagnostic method capable of distinguishing these two diseases. Even within this context, it is important to mention the mixed apnea, called this way by starting as central and then becoming obstructive. It occurs when the breathing movements are restarted at the end of central apnea but the upper airway is obstructed. ${ }^{11}$

The Upper Airway Resistance Syndrome (UARS) is a syndrome of an increase in the upper respiratory tract collapse during sleep, with intermediate values among healthy subjects and with slight or moderate OSAHS. ${ }^{13}$ From the physiological point of view, patients with UARS and with OSAHS are similar, differentiating only by the severity of the airway collapse during sleep. The following symptoms and comorbidities are: Fatigue, insomnia, non-restorative sleep, aching body, headache, depression and hypertension. Both result in awakenings and sleep fragmentation. However, due to differences in epidemiology of these diseases, there is still controversy if the UARS is a separate entity or an early stage of the OSAHS..$^{14}$ As well as the OSAHS, the UARS is debilitating and shows a progressive character, where the majority of patients who have had a diagnosis of UARS and remained untreated during 4 years showed a worsening of the symptoms of insomnia, fatigue and depression, with an expressive increase in prescription drugs, like antidepressants, hypnotics and humor moderators. ${ }^{15}$

\section{DIAGNOSIS AND CLASSIFICATION}

The diagnostic methods used for sleep disturbances investigation range from a subjective evaluation, by means of specific questionnaires, to the daytime or nocturnal polysomnographic or actigraphic records. The nocturnal polysomnography study is the gold standard method for the diagnosis of sleep disorders, registering: Electroencephalogram (EEG), 
electrooculogram (EOG), electromyography (EMG) of mentum and members, oronasal flow volume, thoracoabdominal motion, electrocardiogram (ECG) and pulse oximetry. ${ }^{16}$

The dental surgeon can help diagnosing sleep disturbances referring to a specialist in sleep medicine. A special attention is given to frequent history of morning headaches, a common symptom in $18 \%$ of the snoring or OSAHS patients in comparison to a $5 \%$ in the general population. ${ }^{17}$ Besides, during the clinical examination one can recognize buccal manifestations of OSAHS and snoring in the oropharynx region, tongue, uvula, soft palate and tonsils. ${ }^{18}$

To account these considerations, it is recommended that the size and conditions of the tongue should be evaluated. The Mallampatt index, used by the anesthesiologists to determine the intubation difficulty, may serve as an indicator of air passage obstruction by the tongue volume. It is also known that the tonsils size have a direct relation with OSAHS, once this volume increase can promote reduction of air passage. The observation of shape and volume of uvula and soft palate can not be neglected, as well as the mandibular position, both vertically and horizontally. ${ }^{18}$ One should also evaluate the age, taking into account that muscular tonus decreases with age. It is worth noting the relevance of evaluation the weight, since obesity plays a preponderant role ${ }^{19}$ and contributes to the increase of the cervical circumference. Also in this aspect, it is suggested that hereditary characteristics and biotype be considered, once they are important factors, without necessarily been obese. ${ }^{20}$

Regarding the mandibular posture, radiographic and tomographic images are used to evaluate and to quantify the bone structures of the skull, mandibular and hyoid bone positions. In these images, some soft structures like the tongue and soft palate can be assessed too. When compared to the control group, OSAHS patients presented small and retropositioned mandibles, with subsequent narrowing of the posterior space for the air passage, tongue increment, flaccid soft palate, lower positioning of hyoid bone and retropositioning of the maxilla. ${ }^{20}$

Although its is not the chief complaint, the snoring is the characteristic of OSAHS in children. Breathing difficulties during sleep, headache upon awakening, abundant sweating, excessive thirst upon awakening, nightmares, sleep terror, nocturnal enuresis, little restorative sleep,excessive sleepiness during the day, hyperactivity, attention disorders, poor school performance, behavior disorders, aggressiveness, frequent infections of the airways, frequent otitis and obesity also can be symptoms of OSAHS in children. The most common cause of this disorder in children and adolescents are hypertrophied tonsils and adenoids, but one should also be aware of malformation of the maxilla and / or mandible. In severe cases, pulmonary hypertension and cor pulmonale may be developed. ${ }^{6}$ It is important that the association of apnea with facial dysmorphism calls the expert's attention to an early diagnosis of risk factors for OSAHS and its correct treatment, when dental interventions can be corrective for craniofacial deformations.Nasal obstruction is an initial determining factor in the mouth breathing, and consequently, the change in position of the tongue and teeth in the mouth. Such factors determine functional and structural changes in the face, like hypoplasia of the frontal sinuses, interocular reduction, reduction of the nasal size with collapse of the nasal valve, reduction of the dimensions of the hard palate and consequent reduction of upper arch, leading to a deficient nasal breathing. Mouth breathing, in turn, leads to an increase in volume of the tongue, soft palate and uvula. This frame of facial dysmorphism is called Long Face Syndrome, characterized by a long and narrow face, retrognathia, micrognathia and high and narrow hard palate.

\section{AVAILABLE THERAPIES}

The treatment of OSAHS may involve from simple procedures to complex surgical procedures. The reduction in weight may result in a significant reduction in the frequency of OSAHS and snoring, improvement in the sleep architecture and reduction of excessive daytime sleepiness. ${ }^{19}$

It is well known that alcohol ingestion can cause or exacerbate snoring, increase the frequency and duration of OSAHS episodes, as well as decrease the saturation of oxygen in the blood, ${ }^{21}$ may be caused by the increased upper airway resistance and the reduction of the tonus of the musculature involved. There are reports of increase of the upper airway collapse during sleep in snorers and 
non-snorers individuals, after alcohol ingestion its deleterious effects are directly related to the time elapsed between the ingestion of the drink and the time of the to go to to sleep. Thus, individuals should be advised to not consume alcohol within 3-5 hours before bed time. ${ }^{21}$

There is a consensus in the literature that the CPAP/CFLEX (Continuous Positive Airway Pressure) is the most effective treatment in controlling sleep apnea and on improvement of oxygenation (Fig 1), specially in patients with severe sleep apnea, by generating a continuous or intermittent positive air pressure. ${ }^{5,22}$ Because of its high cost and of the discomfort that comes from its use, it is considered excessive for the treatment of snoring. In addition, patients who use CPAP can present problems in the TMJ's if the mask is used too tight."

The surgical technique - uvulopalatopharyngoplasty, too defended before, showed less effective

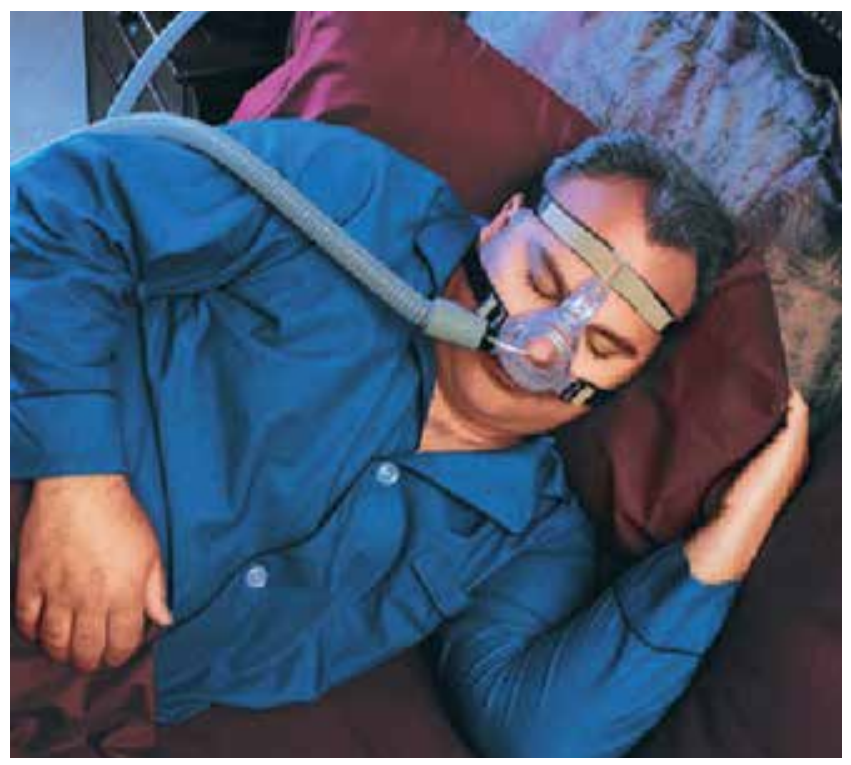

and with more long-term side effects than the use of oral appliances, ${ }^{5,22,23}$ as well as the use of drugs, which have not yet showed sufficient evidence to be recommended for the treatment of obstructive sleep apnea. ${ }^{24}$

Oral appliances are a viable and effective alternative, even when compared to the CPAP in random and controlled clinical trials, ${ }^{25,26,27}$ specially in the treatment of those individuals carrying OSAHS that do not adapt to the use of the apparatus before mentioned. ${ }^{5,22}$ They are usually recommended to patients with slight or moderate OSAHS, however, success in the treatment of severe sleep apnea have already been related. ${ }^{2,27}$ Its indication to teenagers and children still needs a more consistent assessment. ${ }^{5}$

Despite of some advantages over the use of continuous air pressure devices, the indiscriminate use, incorrect or even without any professional follow-up have raised questionings about its indication. ${ }^{5}$

Oral devices operate augmenting the caliber of the upper airways and/or by reducing the obstruction, mostly done in a protrusive position of the mandible, where they may be adjustable or with a preset protrusion amplitude built in its construction (Figs 2, 3 and 4).

In comparison to the effectiveness of the oral devices ( $75 \%$ and $50 \%$ of maximum capacity of protrusion) the ones constructed with a greater mandibular advancement presented the best results. ${ }^{22} \mathrm{An}$ other category of devices are the tongue retainers with its mechanism of action still unknown and are less used than those with mandibular protrusions.

Pain in the temporomandibular joints (TMJs) teeth and muscles, excessive salivation, joint sounds, skeletal and occlusal changes are some of the adverse effects or complications from the use

Figure 1 - Simulation of CPAP usage.

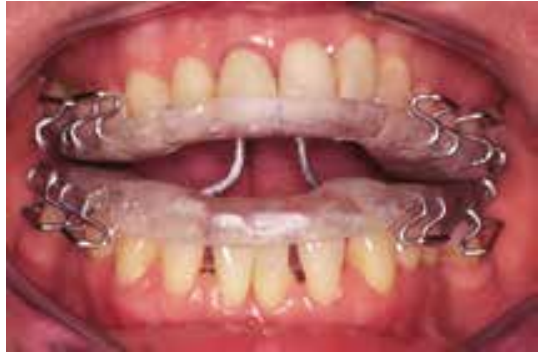

Figure 2 - Oral protrusion device for OSAHS treatment.

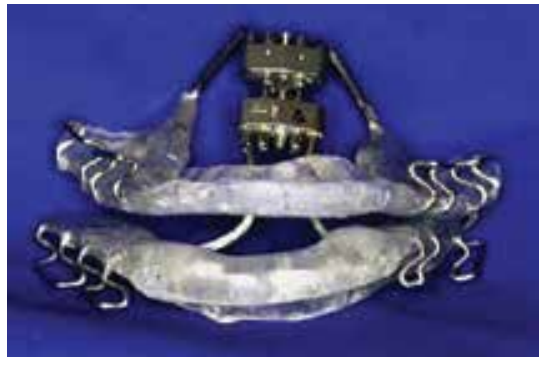

Figure 3 - Adjustable oral protrusion device by means of an expansion screw.

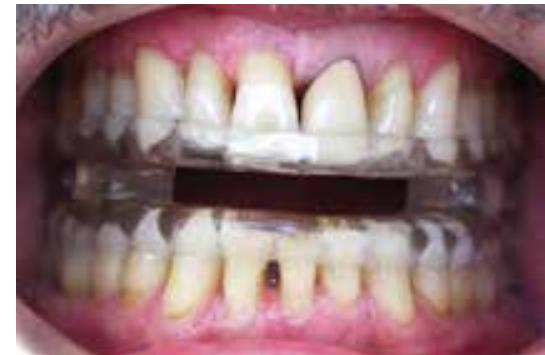

Figure 4 - Oral device with a pre-set protrusion range. 
of oral devices..$^{2,5,22,26,27}$ In some cases, after a period of 8 weeks, these adverse effects have been reported by up to $69 \%$ of the sample ${ }^{25}$ and they seem to be related to the maintenance of a protrusive position of the mandible during long periods of sleep, exerting great stress on the muscles of mastication and TMJs. In the TMJs, it would create a stretch of the retrodiskal ligaments setting off an inflammatory response that could result in arthralgia and joint pain. The musculature would be more susceptible to pain by muscular contraction, spasms or contractures, in addition to tractioning the articular disk anteriorly, which together with the articular ligaments stretch, would increase the possibility of their displacement, causing the onset or exacerbation of articular sounds.

\section{CONDUCT OF TREATMENT PROTOCOL OF OSAHS USING INTRAORAL DEVICES}

The treatment protocol for OSAHS and snoring, using oral devices, recommended by the American Academy of Sleep Medicine, establishes the functions and limitations of activity of physicians and dentists. If, after diagnosis by a qualified physician, the treatment should involve the use of oral devices, the patient will be referred to a dentist, together with clinical informations necessary and/or appropriate, including a copy of the polysomnography and the evaluation of excessive sleepiness. Certainly, this professional must have knowledge related to sleep medicine and the changes arising from alterations in its normal architecture, as well as being familiar with the methods of diagnosis and assessments, including, but not limited to: Polysomnographic examination, excessive sleepiness assessment test and pulse oximetry. The dentist shall then evaluate the possibility of use of oral devices taking into account the conditions of the soft tissues within the mouth, periodontal, dental and articular health, presence of bruxism and possible contra-indications for your its use. ${ }^{28}$ Initial radiographic examination of the teeth and related structures should be requested to facilitate future assessment of possible dental or skeletal changes related to the prolonged use of these devices..$^{27}$ It is also the dentist's role,the choice of the device to be used among the many developed even though comparisons between the different types of oral devices are still limited ${ }^{7,13}$ and their technical or drawing conclusions are not yet possible. ${ }^{22,27}$ After the incorporation of oral device, control and adjustments should be done, as well as monitoring of subjective changes in the disease symptoms. Once satisfactory improvements of symptoms are achieved, the patient should be referenced back to the physician, for a new objective clinical evaluation of the results achieved, including a new polysomnographic examination. It seems a worrying fact that in an American study involving 124 members of the Sleep Disorders Dental Society, where the majority agreed with the statement that only subjective reports of improvement of the symptoms are not sufficient to ensure success in treatment, only in $18 \%$ of cases was carried out a post-treatment polysomnographic examination, even though this same examination was conducted in $95 \%$ of patients during the initial evaluation. ${ }^{29}$

This way, it is our duty to call for a greater commitment of the dental professionals once better results in the treatment of snoring and OSAHS using intraoral devices have been achieved when specialists in sleep medicine and dentists work together effectively.

\section{FINAL CONSIDERATIONS}

The dental surgeon can significantly contribute to identify sleep respiratory disorders, including OSAHS. However, it is strongly disagreed with those who, for the simple identification, suggest some sort of therapy. The diagnosis must, mandatorily, be carried out by a team of medical professionals, and may encompass the following specialties: Otorhinolaryngology, Pulmonology Neurology, Psychiatry and others. For its diagnosis it is crucial an polysomnography examination ${ }^{27}$ and the exclusion of other diseases that can range from simple nasal obstructions and nasal septum deviation, even the presence of tumors and central sleep apnea. Given the exposed, it is clear that OSAHS is multidisciplinary in its etiology and treatment. The authors of this paper emphasize that the diagnosis and treatment must be carried out in an interdisciplinary way and that verification of the diagnosis, as well as the therapy to be applied, must be obligatorily performed by doctor enabled to do so. In other words, although it is the competence of the dental surgeon to identify signs and symptoms of OSAHS, since he is a healthcare professional and as such he 
should be aware of the quality of life of his patients, in addition to perform one of the following types of therapy, that is the inter-occlusal devices, he should not, under no circumstances, indicate that treatment without the request and attestation of indication of it by whom have the right and responsibility to indicate.

The effectiveness and usefulness of oral devices for the treatment of snoring and OSAHS are already well established in the current literature. However, definitive conclusions about their design still aren't as well defined, specially when one ponders about the mandibular protrusive position where they are usually made and its possible implications and undesirable effects, ensuring the need for improvement and/or development of new devices, equally effective and with fewer complications arising from its continued use.

Although already established in the literature a treatment protocol using intraoral devices, establishing the responsibilities of physicians and dentists by giving these professionals a unique opportunity to interact and promote quality of life improvement of these patients, in daily practice it seems that these professionals do not consider this for ignorance or option, contributing in some cases to a less effective treatment.

\section{REFERENCES}

1. Punjabi NM, Welch D, Strohl K. Sleep disorders in regional sleep centers: a national cooperative study. Coleman II Study Investigators. Sleep. 2000 Jun 15;23(4):471-80

2. Gotsopoulos H, Chen C, Qian J, Cistulli PA. Oral appliance therapy improves symptoms in obstructive sleep apnea: a randomized, controlled trial. Am J Respir Crit Care Med. 2002 Sep 1;166(5):743-8.

3. Young T, Palta M, Dempsey J, Skatrud J, Weber S, Badr S. The occurrence of sleep-disordered breathing among middle-aged adults. N Engl J Med. 1993 Apr 29;328(17):1230-5

4. Olson LG, King MT, Hensley MJ, Saunders NA. A community study of snoring and sleep-disordered breathing. Prevalence. Am J Respir Crit Care Med. 1995 Aug;152(2):711-6

5. Ferguson KA, Cartwright R, Rogers R, Schmidt-Nowara W. Oral appliances for snoring and obstructive sleep apnea: a review. Sleep. 2006 Feb 1;29(2):244-62.

6. Young T, Finn L, Hla KM, Morgan B, Palta M. Snoring as part of a dose-response relationship between sleep-disordered breathing and blood pressure. Sleep. 1996 Dec;19(10 Suppl):S202-5

7. Bassetti C, Aldrich MS, Chervin RD, Quint D. Sleep apnea in patients with transient ischemic attack and stroke: a prospective study of 59 patients. Neurology. 1996 Nov:47(5):1167-73.

8. Terán-Santos J, Jiménez-Gómez A, Cordero-Guevara J. The association between sleep apnea and the risk of traffic accidents. Cooperative Group Burgos-Santander. N Engl J Med. 1999 Mar 18;340(11):847-51.

9. Lysdahl M, Haraldsson PO. Long-term survival after uvulopalatopharyngoplasty in nonobese heavy snorers: a 5- to 9-year follow-up of 400 consecutive patients. Arch Otolaryngol Head Neck Surg. 2000 Sep;126(9):1136-40.

10. American Academy of Sleep Medicine. Cost justification for diagnosis and treatment of obstructive sleep apnea. Position statement of the American Academy of Sleep Medicine. Sleep. 2000 Dec 15;23(8):1017-8.

11. Bailey DR, Attanasio R. Dentistry's role in the management of sleep disorders. Recognition and management. Dent Clin North Am. 2001 Oct;45(4):619-30.

12. American Academy of Sleep Medicine. Sleep-related breathing disorders in adults: recommendations for syndrome definition and measurement techniques in clinical research. The Report of an American Academy of Sleep Medicine Task Force. Sleep. 1999 Aug 1;22(5):667-89.

13. Gold AR, Marcus CL, Dipalo F, Gold MS. Upper airway collapsibility during sleep in upper airway resistance syndrome. Chest. 2002 May;121(5):1531-40.

14. Kristo DA, Lettieri CJ, Andrada T, Taylor Y, Eliasson AH. Silent upper airway resistance syndrome: prevalence in a mixed military population. Chest. 2005 May;127(5):1654-7.
15. Guilleminault C, Kirisoglu C, Poyares D, Palombini L, Leger D, Farid-Moayer M, et al. Upper airway resistance syndrome: a long-term outcome study. J Psychiatr Res. 2006 Apr;40(3):273-9.

16. Togeiro SMGP, Smith AK. Métodos diagnósticos nos distúrbios do sono. Rev Bras Psiquiatr. [Internet]. [cited 2011 Jan 3]. Available from: http://www.scielo.br/scielo. php?script=sci_arttext\&pid=S1516-44462005000500003\&lng=en.

17. Ulfberg J, Carter N, Talbäck M, Edling C. Headache, snoring and sleep apnoea. J Neurol. 1996 Sep;243(9):621-5.

18. Bailey DR. Oral evaluation and upper airway anatomy associated with snoring and obstructive sleep apnea. Dent Clin North Am. 2001 Oct;45(4):715-32.

19. Smith PL, Gold AR, Meyers DA, Haponik EF, Bleecker ER. Weight loss in mildly to moderately obese patients with obstructive sleep apnea. Ann Intern Med. 1985 Dec;103(6 ( Pt 1)):850-5.

20. Moran WB, Orr WC. Diagnosis and management of obstructive sleep apnea. Part II. Arch Otolaryngol. 1985 Oct;111(10):650-8

21. Issa FG, Sullivan CE. Alcohol, snoring and sleep apnea. J Neurol Neurosurg Psychiatry. 1982 Apr;45(4):353-9.

22. Lim J, Lasserson TJ, Fleetham J, Wright J. Oral appliances for obstructive sleep apnoea. Cochrane Database Syst Rev. 2006 Jan 25;(1):CD004435.

23. Sundaram S, Bridgman SA, Lim J, Lasserson TJ. Surgery for obstructive sleep apnoea. Cochrane Database Syst Rev. 2005 Oct 19;(4):CD001004.

24. Smith I, Lasserson TJ, Wright J. Drug therapy for obstructive sleep apnoea in adults. Cochrane Database Syst Rev. 2006 Apr 19; (2):CD003002

25. Engleman HM, McDonald JP, Graham D, Lello GE, Kingshott RN, Coleman EL, et al. Randomized crossover trial of two treatments for sleep apnea/hypopnea syndrome: continuous positive airway pressure and mandibular repositioning splint. Am J Respir Crit Care Med. 2002 Sep 15;166(6):855-9.

26. Ferguson KA, Ono T, Lowe AA, Keenan SP, Fleetham JA. A randomized crossover study of an oral appliance vs nasal-continuous positive airway pressure in the treatment of mild-moderate obstructive sleep apnea. Chest. 1996 May;109(5):1269-75.

27. Mehta A, Qian J, Petocz P, Darendeliler MA, Cistulli PA. A randomized, controlled study of a mandibular advancement splint for obstructive sleep apnea. Am J Respir Crit Care Med. 2001 May;163(6):1457-61

28. Petit FX, Pépin JL, Bettega G, Sadek H, Raphaël B, Lévy P. Mandibular advancement devices: rate of contraindications in 100 consecutive obstructive sleep apnea patients. Am J Respir Crit Care Med. 2002 Aug 1;166(3):274-8.

29. Loube MD, Strauss AM. Survey of oral appliance practice among dentists treating obstructive sleep apnea patients. Chest. 1997 Feb;111(2):382-6. 